\title{
Sonic City: Prototyping a Wearable Experience
}

\author{
Ramia Mazé and Margot Jacobs \\ PLAY studio, Interactive Institute \\ Hugo Grauers gata 3, 41296 Göteborg, Sweden \\ \{ramia.maze,margot.jacobs\}@tii.se
}

\begin{abstract}
Sonic City is a project exploring mobile interaction and wearable technology for everyday music creation. A wearable system has been developed that creates electronic music in real-time based on sensing bodily and environmental factors - thus, a personal soundscape is co-produced by physical movement, local activity, and urban ambiance simply by walking through the city.

Applying multi-disciplinary methods, we have developed the wearable from a scenario-driven, aesthetic and lifestyle perspective. A garment has been crafted for 'trying on' interaction and wearabilty options with users on-site in the city. With this prototype, we have been able to explore and rapidly iterate context and content, social and human factors of the wearable application.
\end{abstract}

Keywords: design methods, interaction design, wearable computing, wearability, rapid prototyping, gesture

\section{Introduction}

With advances in ubiquitous computing and mobile applications, technology woven seamlessly into everyday experience and clothing becomes increasingly feasible. With woven circuits, conductive textiles, and miniature sensors, we can start to use technology as a computational material in the same way that we use other, traditional materials in product and fashion design [9]. Application domains for wearables continue to expand, from taskoriented safety-critical applications to leisure-oriented consumer products. As the material and conceptual possibilities expand, it becomes necessary for designers to rethink design processes and prototyping methods to support the development of innovative new user experiences with wearable technology.

In Sonic City, we are a project team developing a wearable system for music creation in our everyday lives and environments. Drawing on gestural interfaces and context-aware computing, we propose an interaction model based on sensor perception of bodily and context factors. If gesture can generally be defined as a conscious or unconscious movement from part of the body, then Sonic City treats mobility through the city as gestural interaction on a larger scale. Thus, the simple act of taking a walk becomes a creative composition, a personal soundscape characterized by our actions, emotions, and the dynamic urban landscape. As a wearable application, music creation is integrated seamlessly into natural behaviors and everyday life.

We have developed a wearable prototype for testing experiential and technical variables in Sonic City. Intended as a personal, expressive and everyday experience, we employ experimental and user-centered methods to design and investigate perceptual, lifestyle, physical, and dynamic variables. Design materials and a wearable garment have been crafted to test and refine options together with users on-site in the city. Here, we present our approach to the design process and interaction, briefly introduce the technical application, and describe initial tests of the wearable prototype that have led to a greater understanding of design for wearable experiences.

\section{Related Issues and Work}

Intended to support personal expression and creativity, our project is inspired by others that deal with cultural and expressive factors. Informed by performance genres and innovative musical interfaces (e.g. [7], [11]), Sonic City intends to take music creation outside the recording studio and performance event and into everyday street life. To this end, Sonic City draws on work in multi-sensor perception and context-awareness (e.g. [3], [5]).

Taking into account issues of social perception and aesthetics dealt with in other wearable projects (e.g. [4]),

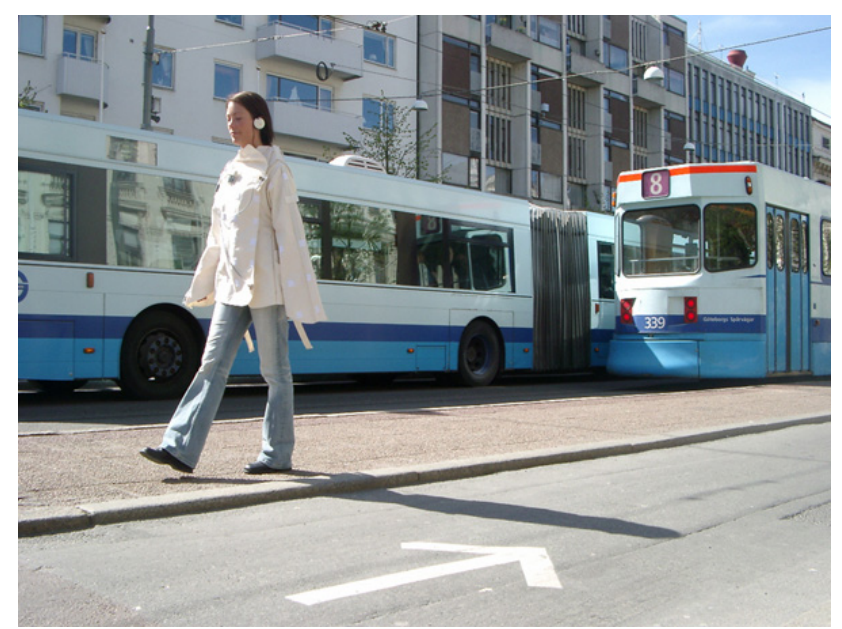

Figure 1. Wearing the Prototype 
our design process brings such issues to bear upon interaction and technical choices. With mobility as the essence of interaction in Sonic City, design factors are heavily concerned with 'wearability' (e.g. [6]). From scenario methods and experience prototyping (e.g. [1], [2]), we have used design materials to approach wearable prototyping experientially.

\section{Our Approach}

In order to better understand, prototype, and design for experience, our process has been highly collaborative and multi-disciplinary. The core team has competence in interaction and product design, engineering, and architecture, and has involved outside experts in sound art, sociology, and cognitive science. To include both experiential and technical perspectives at each stage in the process, design and system development have occurred in parallel. From the technical side, questions and requirements concerned sensing and retrieval, input and control parameters, and sound processing and mapping [5]. From the design side, user-centered design methods have developed these in relation to models for user interaction, wearability, and lifestyle factors.

We believe that in designing for new use contexts and future technology applications, innovative methods are needed to involve users and development teams in considering emerging human factors. Specifically, in Sonic City we had to consider a wide complexity of factors in the user experience, including heterogeneous and multiple use contexts, extended use everyday and over time, and a wide spectrum of user types. Experience prototyping and scenarios have provided vehicles for sharing and decision-making internally and with users.

Our approach to prototype development can be described as 'design and reflection in action'. Staging participatory experiments in the city using design materials, experiential prototypes, and re-configurable features have enabled concept evolution 'in-the-field'. For instance, we have designed and performed initial tests with the prototype encased in a garment designed for modular testing of the technical and experiential variables in the city streets. As a design and research project, Sonic City is a platform for applying innovative design methods for developing an everyday wearable experience.

\subsection{Defining the interaction and system}

At the start of the project, we carried out miniethnographic observations of everyday behavior in the city in order to determine relevant input parameters and implications for context perception. Observations included actions, events, ambiances, and pedestrian's paths as a essentially a composition for structuring these - for example behavioral sequences at crosswalks in terms of glancing, changing course, and speed. Resulting descriptions were broken down into two levels - the body and the environment - and cues that could be used for action and context recognition. From this, we refined the system as a series of wearable sensors for generating music from bodily and environmental activity.

Alongside technical development, we involved users and scenario methods to explore interaction alternatives, perceptions, and design qualities of Sonic City. Written and visual scenarios were developed based on lifestyles, values, and character of people we interviewed. Together with the technical prototype, these were the basis for an in-depth user workshop. This helped us to further define design criteria, system implications, and sound possibilities in terms of a user experience perspective. We decided on a sound mapping balancing foreground/background qualities and explicit/implicit control to support pleasing, dynamic, and comprehensible use everyday.

\section{Wearable Prototype Development}

The Sonic City prototype has been designed to work both experientially and technically. Components of the electronic system are housed in a wearable garment in order 'try on' and rapidly iterate interaction, system, and aesthetic variables. Specifically:

- choice of sensors or combination of sensors

- location of the sensors on the body

- perceived effect of context and action on the music

- options in music processing parameters

- latency and sensitivity in system response

- garment size and shape for movement and mobility

- adjustability and modularity in the wearable design
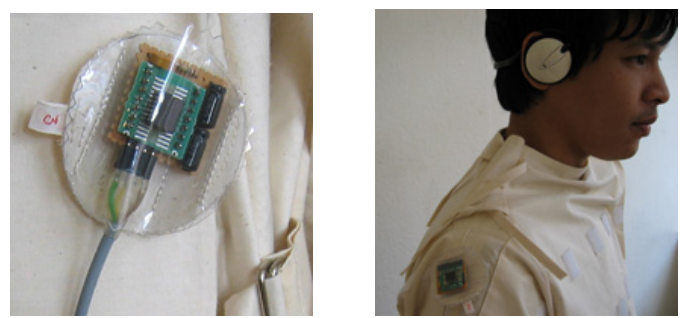

Figures 2 \& 3. Sensor input and sound output

While this prototype has a pivotal role in informing future directions of the project, it is by no means intended as a final form, aesthetic or technology solution. To this end, certain aspects have been deliberately simplified and underplayed, while other variables and options elaborated and highly crafted. Consistent with our approach described earlier, it should be seen as a design material for communicating and testing development questions.

\subsection{Developing the Electronic System}

The technology system in Sonic City [5] has been developed in order to test and iterate significant variables in the experience. Fixed-location hardware components include headphones, a Basic X-24 micro-controller, a 
USB-MIDI converter, and a small laptop running the interactive music programming environment PD.

Variable hardware components include a range of sensors worn on the body, including a light-to-frequency converter, sound gate (microphone), metal detector, accelerometer, thermometer, and pollution sensor. Each extends on a cable from the micro-controller - while not optimal in terms of aesthetics and tidiness, this supports 'plug-and-play'-style connectivity. Thus, individual sensors are easily isolated, combinations quickly tested, and damaged components simply replaced. Additionally, cables provide helpful visual cues as to current sensor configuration when testing multiple options.

As a platform for developing an interaction model and testing the experience outdoors with users, we have prioritized robustness, portability, and modularity in technical decision-making. Ideally, processing would happen in a small, PDA-type device and a future version might be implemented with multiple, miniature sensors wirelessly networked using Smart-Its [10]. For current purposes of discovering and designing for manifold experiential variables, this prototype effectively supports rapid calibration, flexible reconfiguration, and means of trying out multiple options with users in the city.

\subsection{Building the Wearable Form}

A wearable housing for the electronics has been built as a jacket for testing - a sort of Sonic City 'lab coat' for testing in an urban 'wearability lab'. Rather than a design solution, the garment is a sort of working paper prototype. Indeed, even the aesthetic and fabric (an offwhite cotton muslin) were chosen so the jacket could double as a working document: notations of sensor configurations are written directly on the jacket for quick visual reference in user tests and a lasting archive.

Addressing questions of sensor choice, locations, and combinations, we have crafted a modular scheme for sensor configuration. Each sensor is encased in a plastic

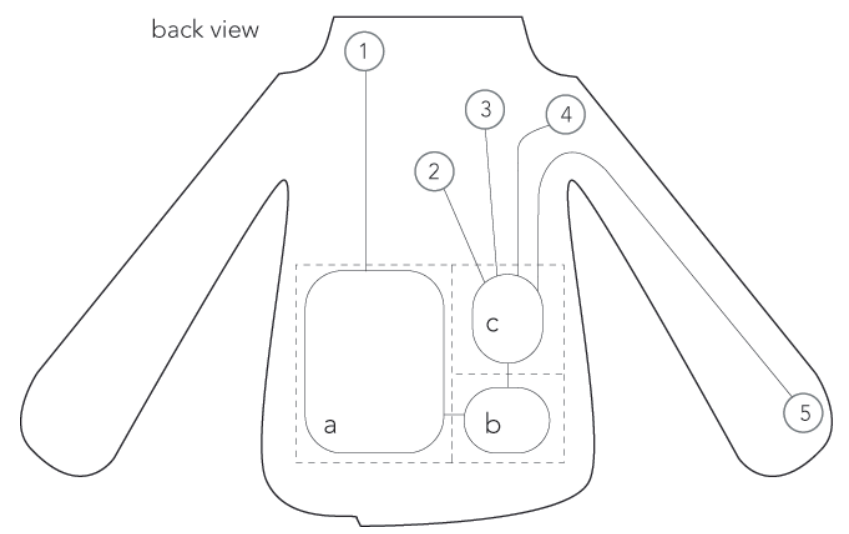

Figure 4. Back view of the jacket showing fixed \& variable placement of electronic components: (a) laptop, (b) USB-MIDI converter, (c) microcontroller, (1) microphone and (2)-(4) sensors patch with Velcro backing and a Velcro grid was sewn on both sides of the garment fabric. Thus, each sensor can be systematically tested nearly anywhere on the upper body. This was particularly important in orienting the accelerometer and microphone to test out variations in the user experience - for instance, the difference in directing the mic toward the feet or the breath.

Dynamic physical movement and mobile factors were of primary factors on designing the form and shape of the garment. A comfortable fit, weight distribution of the electronics, and a wide range of upper-body movement had to be accommodated for users of various shapes and sizes. For maximum maneuverability and comfort, all fixed electronic components were fitted into custom pockets at the back, with Velcro tabs to secure sensor cables along the seams. Adjustable straps and Velcro closures were used to alter the fit of the jacket and ensure a smooth surface so as not to interfere with sensor placement. The arms of the garment were left semi-open at the seam and intentionally elongated to aid with sizing and thermal considerations and it effectively extended sensor placement down to the fingertips. Finally, the garment was sewn as independent, modular pieces to support easy disassembly and reuse.

\subsection{Designing the testing situation}

We have performed initial, in-depth user tests with the wearable prototype in the streets of Göteborg. In order to thoroughly investigate the multiple variables involved, we have designed an interview-style test that was held with two users, in two sites, over two days. Written documentation consists of worksheets to record the path, context factors, sensor placement, and user feedback.

Roles among the project testing team were clearly defined in order to explore as much of the user's experience as possible. One role involved technical calibration and configuration of the hardware and software options. An interviewer gathered and documented user

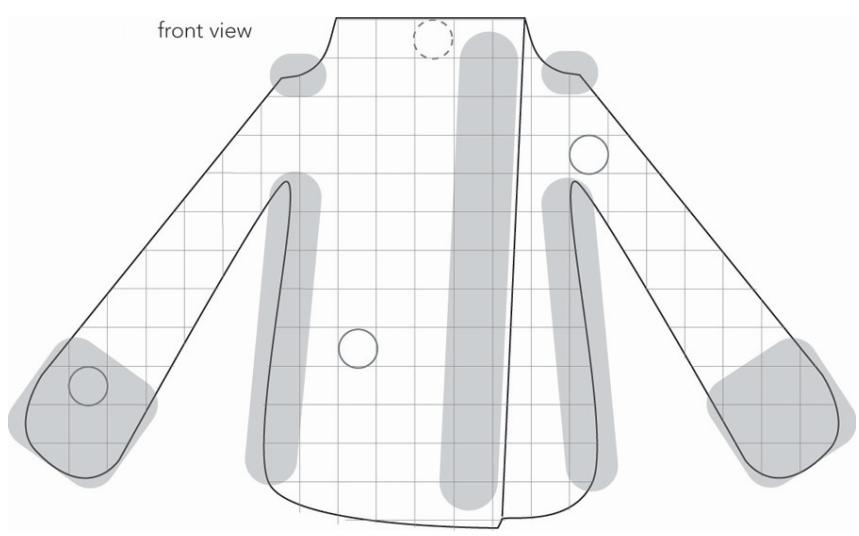

Figure 5. Front view of the jacket showing the pattern for a Velcro grid, a potential sensor configuration, \& re-sizeable areas 
feedback. Two camera people observed and captured the user's behavior and the local environment including reactions of bystanders. The role of analysis took place afterwards, once the recorded musical soundtrack is finally united with video footage and user worksheets.

\section{Initial Results and Future Implications}

Initial results of the user tests have been very positive. Results are primarily qualititative and embedded in experiential feedback though revealing clear implications for the technical side. In terms of the context perception and dynamic mapping strategy, user feedback suggested successful maintenance of interest over the duration of a walk and clear perception of difference between contexts and curiosity about the variable factors. Musical interaction was explored even when standing still - while waiting for a traffic light, one user began improvising physically by swinging his arms and jumping around, smiling spontaneously.

There were also implications for future design directions. There was a good response to flexible sensor placement - for instance, the choice between locating the light sensor on a highly dynamic part of the body such as the hand or a more static location where it might remain shaded and 'muted' for extended periods. These findings point toward potential end-user configurability in the future - this could enable users to customize and refresh the role of Sonic City for their everyday use.

Significant user input will continue to be gathered and these results probed further in future tests, potentially in other cities. We are also planning a 'street-performance' style test in order to learn about general public perceptions and social implications. Future wearable prototype iterations will be developed to reflect emerging criteria and priorities.

In the near future, test results and the user experience scenarios will be incorporated into workshops with fashion and textile designers as vehicles for developing future visions and exploring industrially-relevant or commercially-viable aspects of Sonic City. As we consider increasingly sophisticated iterations, our ongoing challenge will be to develop and apply relevant design and evaluation methods for wearable user experiences.

\section{Acknowledgements}

The core and original project team includes Lalya Gaye (Future Applications Lab, Viktoria Institute), who among many things was responsible for and implemented the technology. We would like to give special thanks to Daniel Skoglund of 8Tunnel2 for contributing to the sound design, Nikolas Makelberge, Daniel Rehn, and Hanna Landin for testing, Magnus Johansson and Sara Lerén for help with methods. This project is funded by the Swedish Foundation for Strategic Research through the Future Applications Lab and the Interactive Institute.
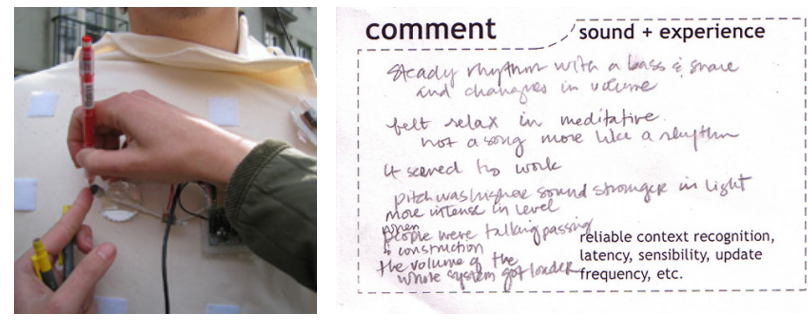

Figures 6 \& 7. Documenting jacket \& worksheet

\section{References}

[1] M. Buchenau and J. Suri, "Experience Prototyping”, Proc. of DIS , ACM Press, 2000, pp. 424-433.

[2] J. Farringdon, A. Moore, N. Tilbury, J. Church, and P. Biemond. "Wearable Sensor Badge \& Sensor Jacket for Context Awareness", Proc. of the $3^{\text {rd }}$ Intl. Symosium. on Wearable Computers. IEEE, 1999, pp. 107-113.

[3] S.K. Feiner, "The Importance of Being Mobile: Some Social Consequences of Wearable Augmented Reality Systems", Proc. of the $2^{\text {nd }}$ Intl. Workshop on Augmented Reality, IEEE and ACM, 1999, pp. 145-148.

[4] L. Gaye, R. Mazé, and L.E. Holmquist, "Sonic City: The Urban Environment as a Musical Interface", forthcoming Proc. of NIME, 2003.

[5] H.W. Gellersen, A. Schmidt, and M. Beigl, "MultiSensor Context-Awareness in Mobile Devices and Smart Artifacts", Mobile Networks and Applications, Kluwer Academic Publishers, MA, USA, 2002, pp. 341-351.

[6] F. Gemperle, C. Kasabach, J. Stivoric, M. Bauer, and R. Martin, "Design for Wearability", Proc. of the $2^{\text {nd }}$ Intl. Symp. on Wearable Computers, IEEE, 1998, pp.116-122.

[7] S. Howard et al. "Endowed props in scenario based design”, Proc.of NordiCHI, 2002, pp. 1-10.

[8] J. Redström, Designing Everyday Computational Things. PhD. Thesis, Gothenburg Studies in Informatics No. 20, Göteborg University, Sweden, 2001.

[9] Smart-Its, information at www.smart-its.org

[10] M. Wanderley and M. Battier, eds., Trends in Gestural Control of Music. IRCAM, Paris, 2000. 